\title{
A validated UHPLC-MS method for tryptophan metabolites: Application in the diagnosis of multiple sclerosis
}

\author{
Ferenc Tömösi ${ }^{a}$, Gábor Kecskeméti ${ }^{a}$, Edina Katalin Cseh ${ }^{b}$, Elza Szabób $^{\mathrm{b}}$, Cecília Rajda ${ }^{\mathrm{b}}$,

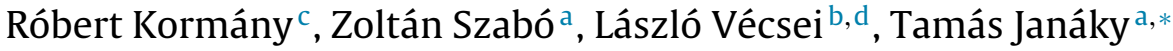 \\ a Department of Medical Chemistry, Interdisciplinary Centre of Excellence, University of Szeged, Dóm tér 8, H-6720, Szeged, Hungary \\ ${ }^{\mathrm{b}}$ Department of Neurology, Interdisciplinary Centre of Excellence, University of Szeged, Semmelweis u. 6, H-6725, Szeged, Hungary \\ ${ }^{\text {c }}$ Egis Pharmaceuticals Plc., Keresztúri út 30-38, H-1106, Budapest, Hungary \\ ${ }^{\mathrm{d}}$ Hungarian Academy of Sciences, MTA-SZTE Neuroscience Research Group, Semmelweis u. 6, H-6725, Szeged, Hungary
}

\section{A R T I C L E I N F O}

\section{Article history:}

Received 12 December 2019

Received in revised form 6 March 2020

Accepted 7 March 2020

Available online 9 March 2020

\section{Keywords:}

Tryptophan metabolism

Derivatization

Liquid chromatography-mass spectrometry

DryLab $^{\circledR} 4$

Validation

Multiple sclerosis

\begin{abstract}
A B S T R A C T
The simultaneous quantitative estimation of tryptophan (TRP) and its metabolites represents a great challenge because of their diverse chemical properties, e.g., presence of acidic, basic, and nonpolar functional groups and their immensely different concentrations in biological matrices. A short ultra high-performance liquid chromatography (UHPLC)-tandem mass spectrometry (MS/MS) method was validated for targeted analysis of TRP and its 11 most important metabolites derived via both kynurenine (KYN) and serotonin (SERO) pathways in human serum and cerebrospinal fluid (CSF): SERO, KYN, 3-hydroxyanthranilic acid, 5-hydroxyindoleacetic acid, anthranilic acid, kynurenic acid (KYNA), 3hydroxykynurenine (3-HK), xanthurenic acid, melatonin, picolinic acid (PICA), and quinolinic acid (QUIN). After selecting the "best" reversed-phase column and organic modifier, DryLab ${ }^{\circledR} 4$ was used to optimize the gradient time and temperature in chromatographic separation. To achieve absolute quantification, deuterium-labeled internal standards were used. Among all compounds, 3 were analyzed in derivatized (butyl ester) forms (3-HK, PICA, and QUIN) and the remaining 9 in underivatized forms. Validation was performed in accordance with the ICH and FDA guidelines to determine the intraday and interday precision, accuracy, sensitivity, and recovery. To demonstrate the applicability of the developed UHPLC-MS/MS method, the aforementioned metabolites were analyzed in serum and CSF samples from patients with multiple sclerosis (multiple sclerosis group) and those with symptomatic or noninflammatory neurological diseases (control group). The concentration of QUIN dramatically increased, whereas that of KYNA slightly decreased in the multiple sclerosis group, resulting in a significantly increased QUIN/KYNA ratio and significantly decreased PICA/QUIN ratio.
\end{abstract}

(c) 2020 Elsevier B.V. All rights reserved.
Abbreviations: 3-HANA, 3-hydroxyanthranilic acid; 3-HK, 3hydroxykynurenine; 5-HIAA, 5-hydroxyindoleacetic acid; aCSF, artificial cerebrospinal fluid; AGC, automatic gain control; ANA, anthranilic acid; CSF, cerebrospinal fluid; EDSS, expanded disability status scale; FA, formic acid; FD, fluorescence detector; GC, gas chromatography; HPLC, high-performance liquid chromatography; HQC, high-level quality control; KAT, kynurenine aminotransferase; KP, kynurenine pathway; KYN, L-kynurenine; KYNA, kynurenic acid; LOD, limit of detection; LOQ limit of quantification; LQC, low-level quality control; MELA, melatonin; MQC, medium-level quality control; MS/MS, tandem mass spectrometry; PFP, pentafluorophenyl; PICA, picolinic acid; PRM, parallel reaction monitoring; QC, quality control; QUIN, quinolinic acid; RPC, reversed-phase chromatography; RRMS, relapsing-remitting multiple sclerosis; RSD, relative standard deviation; SERO, serotonin; SIL-IS, stable isotope-labeled internal standard; TRP, tryptophan; UHPLC, ultra high-performance liquid chromatography; XA, xanthurenic acid.

* Corresponding author.

E-mail address: janaky.tamas@med.u-szeged.hu (T. Janáky).

\section{Introduction}

The kynurenine (KYN) pathway (KP) is the major metabolic pathway of the essential amino acid tryptophan (TRP), which leads to the production of neuroprotective and neurotoxic compounds. Changes in the concentrations of neuroprotectants such as kynurenic acid (KYNA) and picolinic acid (PICA) have been described in different diseases, but the excitotoxin quinolinic acid (QUIN) and the free radical generator 3-hydroxykynurenine (3-HK) are associated with different neurodegenerative diseases including multiple sclerosis [1]. KYNA is an endogenous glutamate receptor antagonist that affects all ionotropic glutamate receptors including NMDA, kainate, and AMPA receptors, and it exhibits the highest affinity for the NMDA receptor and displays antioxidant and free radical-scavenging activities [2]. Conversely, QUIN is known 
for its broad-spectrum neurotoxic effects including its role as an NMDA receptor agonist, in addition to its roles in antioxidant depletion, lipid peroxidation, and oxygen intermediate generation [1].

Multiple sclerosis is a disabling autoimmune, inflammatory, neurodegenerative, demyelinating disease affecting the central nervous system. The diagnosis of multiple sclerosis is based on clinical symptoms suggestive of demyelinating disorders as proven by MRI and lumbar puncture. In addition to dissemination of the disease in space and time, it is essential to exclude other diseases. According to McDonald's criteria, as revised in 2017, oligoclonal gammopathy in cerebrospinal fluid (CSF) or the simultaneous presence of gadolinium-enhancing and nonenhancing lesions can verify dissemination in time. Dissemination in space can be proven by the presence of one or more T2 lesions in the periventricular, cortical, juxtacortical, or infratentorial space or spinal cord [3]. Multiple sclerosis is considered active if the following findings are present: relapse, confirmed expanded disability status scale (EDSS) progression, new/enlarged MRI lesions, and disease-related brain atrophy [4].

Recently, multiple sclerosis subtypes were differentiated according the concentrations of the different TRP metabolites [1,5] because changes in their concentrations appear to serve as potential biomarkers. For example, in acute relapse of relapsing-remitting multiple sclerosis (RRMS), increased neurotoxic QUIN concentration, higher QUIN/KYN and QUIN/KYNA ratios [6], and lower KYNA concentration can be observed in CSF, whereas increased KYNA concentration were found in plasma. The expression of KYN aminotransferase (KAT) also increased in red blood cells. Decreased TRP concentration in both serum and CSF were described in patients with RRMS compared with those in control subjects. Interestingly, during remission, lower KYNA concentration can be observed in CSF [7], whereas higher KYNA concentration can be found in patients with the progressive form of multiple sclerosis [8].

The simultaneous quantitative estimation of TRP and its metabolites represents a great challenge because of their diverse chemical properties, namely, presence of acidic, basic, and nonpolar functional groups and their immensely different concentrations in biological matrices such as serum (plasma) and CSF. Numerous chromatographic methods have been developed for the successful quantitative estimation of these metabolites using gas chromatography (GC), high-performance liquid chromatography (HPLC), ultra high-performance liquid chromatography (UHPLC) coupled with a fluorescence detector (FD), a UV detector, or tandem mass spectrometry (MS/MS) [8-11]. The advantages of UHPLC-MS/MS include its sensitivity, ability to quantitate multiple compounds in a single run, short elution time, excellent separation efficiency, and requirement of small sample amount. Hényková et al. [9] developed a UHPLC-MS/MS method for the quantitative estimation of TRP, KYN, 3-HK, KYNA, 3-hydroxyanthranilic acid (3-HANA), anthranilic acid (ANA), serotonin (SERO), melatonin (MELA), and other important neuroactive metabolites derived from TRP in human CSF and serum. Fuertig et al. [10] described the successful quantitative estimation of 13 compounds related to TRP in the brains and plasma of mice and CSF and plasma of nonhuman primates. However, none of these studies considered the separation and peak shape of early eluting components. Because of the different chemical characteristics of TRP and its metabolites, the combination of several methods was used to measure their concentrations by Guillemin et al. [8] including UHPLC coupled with a diode array detector and an FD were used to measure TRP, KYN, 3-HK, 3-HANA, and ANA concentrations using isocratic separation. In addition, gradient elution was performed to measure KYNA concentration using an FD, and GC coupled with a mass spectrometer was used to measure PICA and QUIN concentrations.
The main purpose of the current study was to develop a new, robust UHPLC-MS/MS method to quantify the concentrations of TRP and its 11 most important metabolites (Fig. 1), which were derived via both KP and SERO pathways, including the concentrations of the rarely measured metabolites PICA and QUIN. Optimization of a chromatographic method can be supported and accelerated using in silico simulation software [12] to define the most appropriate conditions for rapid, sensitive, precise, and reproducible analysis as economically as possible. After column and organic modifier scouting, the DryLab ${ }^{\circledR} 4$ method development/optimization software [13] was applied to predict retention and resolution as functions of gradient time $\left(t_{G}\right)$ and temperature (T).

To the best of our knowledge, this is the first report on the simultaneous quantitative characterization of endogenous SERO, KYN, 3-HANA, TRP, 5-hydroxyindoleacetic acid (5-HIAA), ANA, KYNA, 3HK, xanthurenic acid (XA), MELA, PICA, and QUIN in serum and CSF of patients with multiple sclerosis using UHPLC-MS/MS.

\section{Materials and methods}

\subsection{Reagents and chemicals}

All reagents and chemicals were of analytical or LC-MS grade. TRP and its metabolites d4-PICA and $n$-butanol were purchased from Sigma-Aldrich (St. Louis, MO, USA). d3-3-HK was obtained from Buchem B. V. (Apeldoorn, The Netherlands). Other deuterated internal standards (ISs; d4-SERO, d4-KYN, d3-3-HANA, d5-TRP, d5-5-HIAA, d5-KYNA, d4-XA, d4-MELA, and d3-QUIN) were purchased from Toronto Research Chemicals (Toronto, ON, Canada). Acetonitrile (ACN), methanol $(\mathrm{MeOH})$, water, HPLC-grade ammonium formate, and ammonium acetate were obtained from VWR Chemicals (Monroeville, PA, USA). Formic acid (FA) was purchased from Fisher Scientific (Portsmouth, NH, USA) and acetyl chloride from Alfa Aesar (Haverhill, MA, USA).

\subsection{Preparation of standard, IS, and quality control (QC) solutions}

Stock solutions were prepared individually at a final concentration of $1 \mathrm{mg} / \mathrm{mL}$, except 3-HK and d3-3-HK $(0.5 \mathrm{mg} / \mathrm{mL})$. According to their stability and solubility, several solvents were used. SERO, d4-SERO, TRP, d5-TRP, ANA, PICA, and d4-PICA were dissolved in water-MeOH-FA-ascorbic acid (50:50:0.1:0.02, v/v/v/v). KYN, d4KYN, 5-HIAA, d5-5-HIAA, 3-HK, d3-3-HK, MELA, d4-MELA, QUIN, and d3-QUIN were dissolved in $\mathrm{MeOH}$ containing $0.1 \%(\mathrm{v} / \mathrm{v}) \mathrm{FA}$ and $0.02 \%(v / v)$ ascorbic acid. 3-HANA, d3-3-HANA, KYNA, d5-KYNA, $\mathrm{XA}$, and $\mathrm{d} 4$-XA were dissolved in dimethyl sulfoxide. All standard stock solutions were prepared on ice, divided into $200-\mu \mathrm{L}$ aliquots, and stored at $-80^{\circ} \mathrm{C}$ until further use.

Calibration standards were prepared at 12 levels and QC samples at three levels (low-level QC [LQC], eighth point of calibration; middle-level QC [MQC], fourth point of calibration; and high-level QC [HQC], second point of calibration) in artificial CSF (aCSF; containing $127 \mathrm{mM} \mathrm{NaCl}, 1.0 \mathrm{mM} \mathrm{KCl}, 1.2 \mathrm{mM}$ $\mathrm{KH}_{2} \mathrm{PO}_{4}, 26 \mathrm{mM} \mathrm{NaHCO}, 10 \mathrm{mM}$ D-glucose, $2.4 \mathrm{mM} \mathrm{CaCl}_{2}, 1.3 \mathrm{mM}$ $\mathrm{MgCl}_{2}$, and $5.26 \mu \mathrm{M}$ bovine serum albumin) [14] for CSF analysis. Calibration standards comprised $250 \mu \mathrm{L}$ aCSF, $20 \mu \mathrm{L}$ standard solution mix (31.25-1000 nM SERO, 6.25-200 nM KYN, 0.25-8 nM 3-HANA, 250-8000 nM TRP, 12.5-400 nM 5-HIAA, 0.6-20 nM ANA, 0.25-8 nM KYNA, 1.25-40 nM 3-HK, 0.1-2 nM XA, 0.25-8 nM MELA, 2-60 nM PICA, and 5-160 nM QUIN in $0.1 \%$ [v/v] aqueous FA), and $910 \mu \mathrm{L}$ ice-cold ACN containing $10 \mu \mathrm{L}$ stable isotope-labeled (SIL)-IS mix (800 nM d4-SERO, 20 nM d4-KYN, 3 nM d3-3-HANA, 4000 nM d5-TRP, 200 nM d5-5-HIAA, 2 nM d5-KYNA, 8 nM d33-HK, $0.4 \mathrm{nM}$ d4-XA, $4 \mathrm{nM}$ d4-MELA, $15 \mathrm{nM}$ d4-PICA, and $25 \mathrm{nM}$ d3-QUIN). 


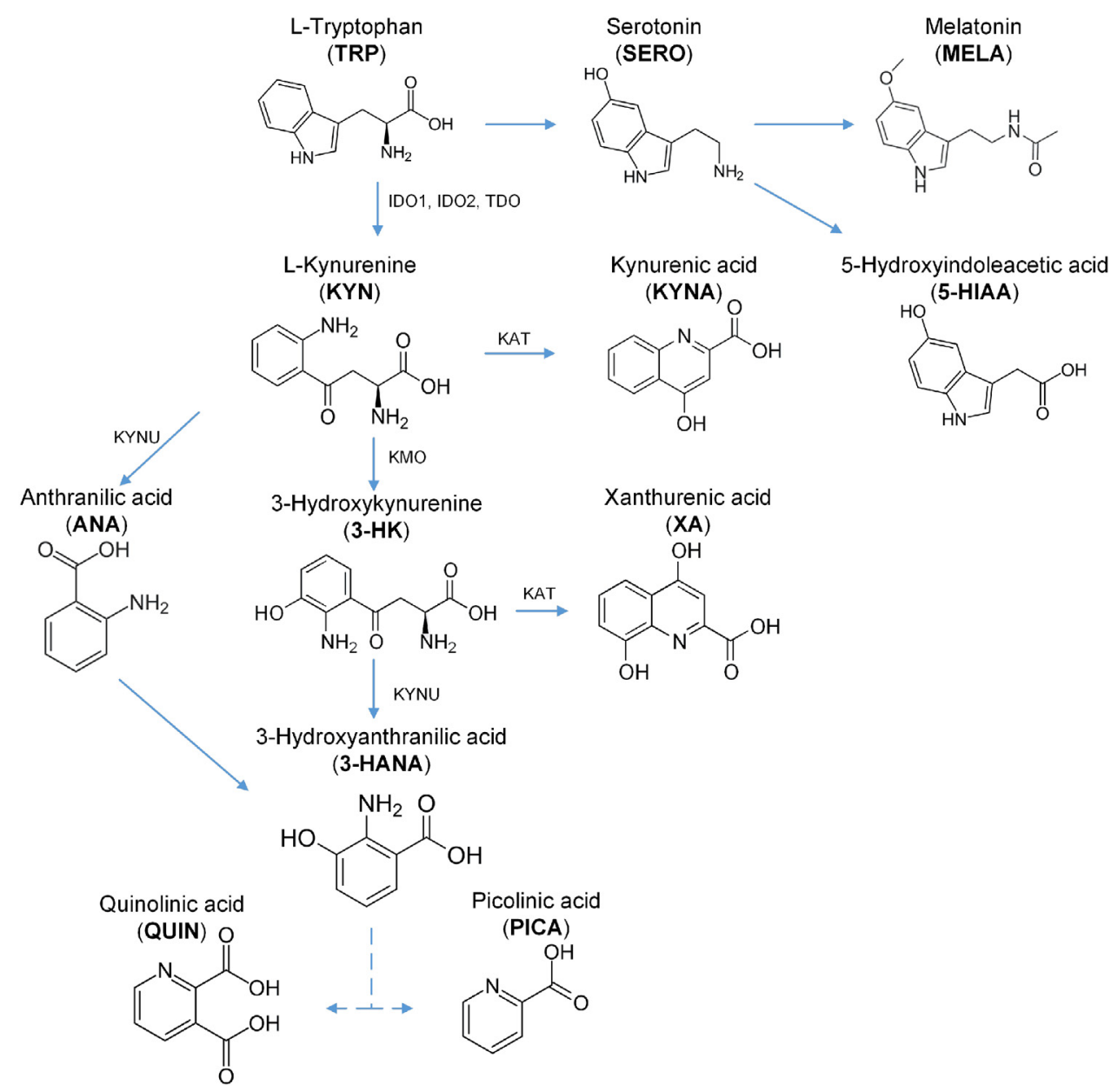

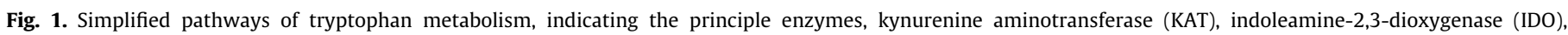
tryptophan-2,3-dioxygenase (TDO), kynureninase (KYNU), and kynurenine-3-monooxygenase (KMO).

The calibration standards and QC samples for serum analysis were prepared in charcoal-stripped human serum, which was prepared as described by Möller et al. [15]. Briefly, $1.2 \mathrm{~g}$ of charcoal-activated powder (Fisher Scientific) was added to $20 \mathrm{~mL}$ serum, rotated for $2 \mathrm{~h}$, and centrifuged at $14,000 \times g$ for $10 \mathrm{~min}$ to obtain the supernatant. Then, "blank" serum was confirmed by LC-MS/MS to be free of TRP and its metabolites. Calibration standards comprised $100 \mu \mathrm{L}$ "blank" serum, $10 \mu \mathrm{L}$ standard solution mix [156.25-5000 nM SERO, 312.5-10000 nM KYN, 7.8-250 nM 3HANA, 6.25-200 $\mu$ M TRP, 7.8-250 nM 5-HIAA, 6.25-200 nM ANA, 4.7-150 nM KYNA, 6.25-200 nM 3-HK, 1.5-50 nM XA, 0.16-5 nM MELA, 3.125-100 nM PICA, and 62.5-2000 nM QUIN in $0.1 \%(\mathrm{v} / \mathrm{v})$ aqueous FA], and $370 \mu \mathrm{L}$ ice-cold acetone: $\mathrm{MeOH}(1: 1, \mathrm{v} / \mathrm{v})$ containing $10 \mu \mathrm{L}$ SIL-IS mix (1500 nM d4-SERO, $1000 \mathrm{nM}$ d4-KYN, $65 \mathrm{nM}$ d3-3-HANA, 5250 nM d5-TRP, 200 nM d5-5-HIAA, 50 nM d5-KYNA, $90 \mathrm{nM}$ d3-3-HK, $25 \mathrm{nM}$ d4-XA, $4 \mathrm{nM}$ d4-MELA, $80 \mathrm{nM}$ d4-PICA, and $300 \mathrm{nM}$ d3-QUIN). Concentrations were selected in accordance with endogenous analyte concentrations and to reach a proper signal to noise ratio.

\subsection{Collection of human CSF and serum samples}

The females included in this study, who underwent both lumbar puncture and blood sample collection, were enrolled at the Department of Neurology, University of Szeged. Approval for the human study was granted by the local Ethical Committee of the University of Szeged (46/2014 and 143/2015), and the study protocol adhered to the tenets of the most recent revision of the Decla-
Table 1

Detailed demographic and clinical data of the study population.

\begin{tabular}{lll}
\hline Subject & Multiple sclerosis group & Control group \\
\hline Age in years $($ mean \pm SD) & $33.84 \pm 9.14$ & $37.57 \pm 10.09$ \\
Age at onset $($ mean \pm SD) & $32.47 \pm 9.00$ & - \\
EDSS (median, IQR) & $0.5(0,1.5)$ & -
\end{tabular}

Data are presented as the mean \pm SD or median and interquartile range for EDSS. EDSS, expanded disability status scale; IQR, interquartile range; SD, standard deviation.

ration of Helsinki for experiments involving humans. All enrolled subjects provided voluntary signed informed consent for participation. Inclusion criteria for the multiple sclerosis group were older than 18 years, female sex, diagnosis of RRMS, clinical follow-up for at least 2 years, and availability of serum and CSF samples at the biobank at the time of diagnostic lumbar puncture $(n=20)$. The demographic and clinical data of the cohort are presented in Table 1. Disability was quantitatively estimated using EDSS. EDSS values ranged between 0 and 5.5 (median $=0.5$ ). Age-matched patients $(\mathrm{n}=14)$ with symptomatic neurological disorder (e.g., headache) or noninflammatory neurological disease (e.g., benign intracranial hypertension) who had been followed up for at least 2 years served as the control group.

CSF samples were centrifuged immediately after lumbar puncture at $3500 \mathrm{rpm}$ for $10 \mathrm{~min}$, and aliquots of $500 \mu \mathrm{L}$ were stored at $-80^{\circ} \mathrm{C}$ until further use. Whole-blood samples were collected in Vacutainer tubes, centrifuged, and the supernatant stored as mentioned previously. Serum and CSF samples were collected and 


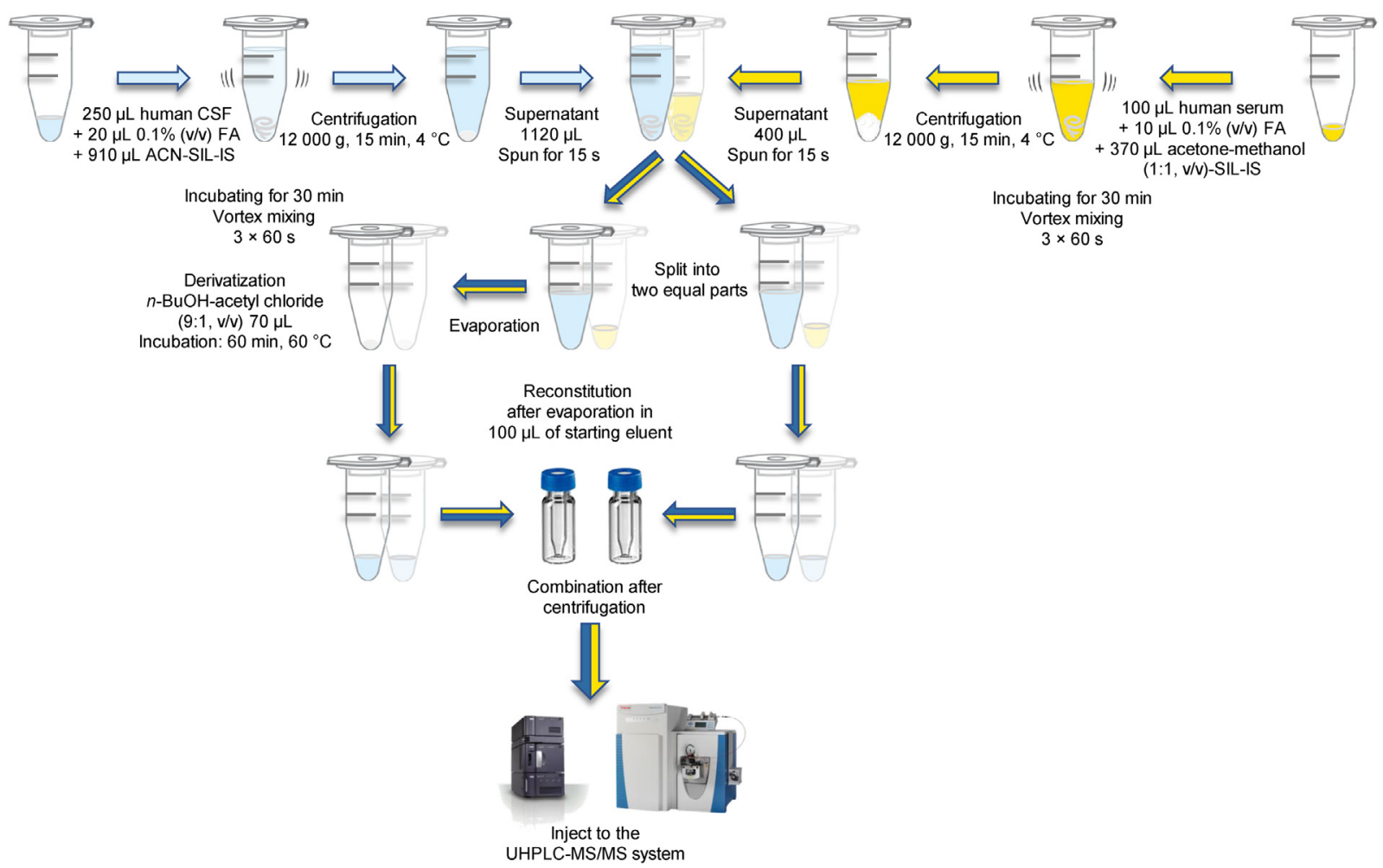

Fig. 2. Flow chart of the sample preparation process of human CSF and serum samples.

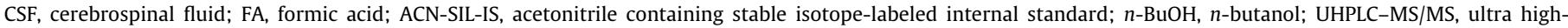
performance liquid chromatography-tandem mass spectrometry.

stored according to the standardized international biobanking consensus protocol of the BIOMS-Eu network [16].

\subsection{Preparation of human CSF and serum samples for analysis}

Prior to profiling the KP and SERO pathways, samples were relabeled; therefore, a blind study was conducted. To $250 \mu \mathrm{L}$ of each CSF sample, $20 \mu \mathrm{L} 0.1 \%$ (v/v) of aqueous FA and $910 \mu \mathrm{L}$ ice-cold ACN containing $10 \mu \mathrm{L}$ SIL-IS mix (the same as used in the preparation of the calibration standards) were added, and the mixture was vortexed for $30 \mathrm{~s}$. It was allowed to rest for $15 \mathrm{~min}$ at $-20^{\circ} \mathrm{C}$, vortexed for $60 \mathrm{~s}$ to support protein precipitation, and incubated for another $15 \mathrm{~min}$ at $-20^{\circ} \mathrm{C}$. The supernatant was obtained via centrifugation of the mixture for $15 \mathrm{~min}$ at $12,000 \times \mathrm{g}$ at $4{ }^{\circ} \mathrm{C}$. The supernatant $(1120 \mu \mathrm{L})$ was transferred to a new tube, centrifuged for $15 \mathrm{~s}$, and split into two equal parts. After concentration under vacuum (Savant SC 110 A Speed Vac Plus, Savant, USA), half of the sample was treated with $70 \mu \mathrm{L}$ derivatizing reagent (n-butanolacetyl chloride, $9: 1, \mathrm{v} / \mathrm{v}$ ) and incubated for $1 \mathrm{~h}$ at $60^{\circ} \mathrm{C}$. The mixture was dried under nitrogen before reconstitution. Both parts of the sample were dissolved in $100 \mu \mathrm{L}$ starting eluent, vortexed, centrifuged, and combined (Fig. 2).

Serum samples were prepared in the same manner, except that to $100 \mu \mathrm{L}$ of serum sample were mixed with $10 \mu \mathrm{L} 0.1 \%(\mathrm{v} / \mathrm{v})$ aqueous FA and $370 \mu \mathrm{L}$ ice-cold acetone-MeOH $(1: 1, \mathrm{v} / \mathrm{v})$ containing $10 \mu \mathrm{L}$ SIL-IS mix, and $400 \mu \mathrm{L}$ supernatant was processed further (Fig. 2).

\subsection{Instrumentation and UHPLC-MS/MS analysis}

\subsubsection{Development and optimization of UHPLC separation}

UHPLC separation of TRP and its metabolites was performed on an ACQUITY I-Class UPLC ${ }^{\mathrm{TM}}$ liquid chromatography system (Waters, Manchester, UK) comprising Binary Solvent Manager,
Sample Manager-FL, and Column Manager. The dwell volume of the system was $100 \mu \mathrm{L}$.

To select the most appropriate column for analyte separation, the following reversed-phase columns were tested: BEH C18 $2.1 \times 50 \mathrm{~mm}(1.7 \mu \mathrm{m})$ and Cortecs $\mathrm{C} 182.1 \times 50 \mathrm{~mm}(1.6 \mu \mathrm{m})$ from Waters (Milford, MA, USA) and Kinetex C18 $2.1 \times 100 \mathrm{~mm}(2.6 \mu \mathrm{m})$; Kinetex EVO C18 $2.1 \times 150 \mathrm{~mm}(5.0 \mu \mathrm{m})$; Luna C18 $2.0 \times 100 \mathrm{~mm}$ $(2.5 \mu \mathrm{m})$; Luna Omega Polar C18 $2.1 \times 50 \mathrm{~mm}$, $(1.6 \mu \mathrm{m})$; Luna Omega PS C18 $2.1 \times 50 \mathrm{~mm}(1.6 \mu \mathrm{m})$, Kinetex C8 $2.1 \times 150 \mathrm{~mm}$ $(2.6 \mu \mathrm{m})$, Jupiter Proteo C12 $2.0 \times 150 \mathrm{~mm}(4.0 \mu \mathrm{m})$, and Luna Phenyl-Hexyl $2.1 \times 100 \mathrm{~mm}(2.6 \mu \mathrm{m})$ from Phenomenex (Torrance, CA, US).

Chromatograms were developed with a generic MS-compatible gradient from $2 \%$ to $80 \% \mathrm{~B}$ in 7 min using $0.1 \%$ FA as solvent $\mathrm{A}$ and $0.1 \% \mathrm{FA}$ in $\mathrm{MeOH}$ as solvent $\mathrm{B}$. Similar gradient elution was performed using ACN in solvent $B$ as an organic modifier. For each measurement, the flow rate was set at $300 \mu \mathrm{L} / \mathrm{min}$ and $\mathrm{T}$ at $25^{\circ} \mathrm{C}$. In total, $20 \mu \mathrm{L}$ of a standard mixture containing $2 \mu \mathrm{g} / \mathrm{mL}$ of each analyte was injected into the columns. The UHPLC system was controlled using MassLynx 4.1 SCN 901 (Waters).

To optimize separation on the "best" column, a 2D retention model was built on the basis of four chromatographic runs after injection of a mixture of all analytes. The $t_{G}$ values were set at 5 and 15 min to linearly change the eluent composition from 10 $\%$ to $90 \% \mathrm{MeOH}$ containing $0.1 \% \mathrm{FA}$. Chromatography was performed at $25^{\circ} \mathrm{C}$ and $50^{\circ} \mathrm{C}$ at a flow rate of $300 \mu \mathrm{L} / \mathrm{min}$. Peaks in different chromatograms were identified by detecting the molecular ions of every analyte on a mass spectrometer. The resulting chromatographic data (retention time, peak width, and area) were entered into DryLab ${ }^{\circledR} 4$ (Molnár-Institute, Berlin, Germany) to create a $2 \mathrm{D}$ resolution map displaying the critical resolutions of the peaks separated against $\mathrm{t}_{\mathrm{G}}$ and $\mathrm{T}$.

Chromatographic separation for quantitative estimation of TRP and its 11 metabolites in CSF and serum was performed at 
$25^{\circ} \mathrm{C}$ on a pentafluorophenyl (PFP) column (Phenomenex; $100 \AA$, $100 \mathrm{~mm} \times 2.1 \mathrm{~mm}$, particle size $2.6 \mu \mathrm{m}$; "best" column) protected by a PFP guard column (Phenomenex) using $0.1 \%(\mathrm{v} / \mathrm{v})$ aqueous FA as solvent $\mathrm{A}$ and $\mathrm{MeOH}$ containing $0.1 \%(\mathrm{v} / \mathrm{v}) \mathrm{FA}$ as solvent $\mathrm{B}$. The final gradient was set as follows: $0.0 \mathrm{~min}, 10 \% \mathrm{~B} ; 1.0 \mathrm{~min}, 30 \% \mathrm{~B}$; $3.0 \mathrm{~min}, 50 \% \mathrm{~B}$; $3.5 \mathrm{~min}, 90 \% \mathrm{~B} ; 5.0 \mathrm{~min}, 90 \% \mathrm{~B} ; 5.1 \mathrm{~min}, 10 \% \mathrm{~B}$; and $7 \mathrm{~min} 10 \% \mathrm{~B}$. For each measurement, the flow rate was set at $300 \mu \mathrm{L} / \mathrm{min}$. Sample T was maintained at $5^{\circ} \mathrm{C}$. Finally, $20 \mu \mathrm{L}$ of the sample was injected into the UHPLC-MS/MS system.

\subsubsection{Optimization of mass spectrometric analysis}

All mass spectrometric measurements were conducted using the QExactive ${ }^{\mathrm{TM}}$ Plus Hybrid Quadrupole-Orbitrap Mass Spectrometer (Thermo Fisher Scientific, San Jose, CA, USA) connected online to the UHPLC instrument. The instrument was operated in the positive-ion mode using the equipped HESI-II source with the following parameters: capillary $\mathrm{T}, 256^{\circ} \mathrm{C}$; spray voltage, $3.5 \mathrm{kV}$; aux gas heater $\mathrm{T}, 406^{\circ} \mathrm{C}$; sheath gas flow, 48 ; aux gas flow, 11 ; sweep gas flow, 2; and S-lens RF level, 50.0 (source auto-defaults). Full scan was conducted with a mass range of $50-300 \mathrm{~m} / \mathrm{z}$ and resolution of 17,500 . The automatic gain control (AGC) setting was defined as $3 \times 10^{6}$ charges, and the maximum injection time was set to $60 \mathrm{~ms}$.

For quantitative mass spectrometric analysis of TRP and its metabolites using MS/MS, the parallel reaction monitoring (PRM) data acquisition mode was selected. To reach the best precursor/product transition for quantitation and maximize sensitivity, the optimal fragmentation conditions and collision energies of each analyte were identified. This optimization procedure was performed for each individual standard by directly infusing each solution into the ion source using a Hamilton syringe at a flow rate of $20 \mu \mathrm{L} / \mathrm{min}$.

The concentrations of TRP and its metabolites in biological samples were measured by monitoring the appropriate transitions (Table 2) determined previously. AGC target was set at $5 \times 10^{6}$ charges for TRP and d5-TRP because of their higher concentrations and $2 \times 10^{5}$ charges for the remaining analytes. The maximum injection time was set at $60 \mathrm{~ms}$ and resolution at 17,500. A divert valve placed after the analytical column was programmed to switch flow onto the mass spectrometer only when analytes of interest eluted from the column (1.4-5.0 min) to prevent excessive contamination of the ion source and ion optics. The washing procedures of the autosampler before and after injecting samples were programmed to avoid carryover of analytes.

Control of the mass spectrometer, data acquisition, and data processing was conducted using Xcalibur ${ }^{\mathrm{TM}} 4.1$ (Thermo Fisher Scientific).

\subsection{Method validation}

Biological matrices devoid of TRP and its metabolites are not readily available; hence, surrogate matrices (aCSF and "blank" serum) were used to demonstrate the efficiency of the method. In this study, SIL-IS analogs were used to validate the UHPLC-MS/MS method. For validation, the linearity, limits of detection (LOD) and quantification (LOQ), precision, accuracy, and recovery were assessed following the ICH and FDA guidelines [17,18].

\subsubsection{Linearity, $L O D$, and $L O Q$}

The calibration curves of the 12 analytes were constructed from the peak area ratios of the compound to SIL-IS at 11 levels using mixed working standard solutions. According to the acceptance criteria, the calibration curve should have a correlation coefficient $\left(\mathrm{r}^{2}\right)$ of 0.99 or better. The LOD and LOQ of each analyte were calculated based on the standard error of the intercept. LOD and LOQ were calculated using the formulas $3.3 \times \alpha / \mathrm{S}$ and $10 \times \alpha / \mathrm{S}$, respectively, where $\alpha$ is the standard error of the $y$-intercept and S is the slope of the calibration curve (Supplementary Tables 1-2).

\subsubsection{Precision and accuracy}

The accuracy and intraday and interday precisions were calculated by determining five replicates of LQC, MQC, and HQC samples over 3 consecutive days $(n=45)$. The concentration values of QCs are presented in Supplementary Tables 3-6.

Regarding precision, the acceptance criterion was $\pm 15 \%$ with respect to relative standard deviation (RSD). Regarding accuracy, the limit was the same relative to definite concentrations.

\subsubsection{Recovery}

To determine recovery, two batches were prepared as described previously [19]. The first batch was spiked before protein precipitation, whereas the second batch was spiked after protein precipitation. To achieve reliable results, five LQC, MQC, and HQC replicates were prepared (see Chapter 2.2). The recovery of TRP and its metabolites was estimated by comparing the calculated concentration ratios of the two batches at three QC levels (LQC, MQC, and HQC; $\mathrm{n}=45$ ).

\subsection{Statistical analysis}

The calculation of peak area ratios and the calibration and quantitation of analytes were performed from collected raw data using Xcalibur ${ }^{\mathrm{TM}}$ Quan Browser (Thermo Fisher Scientific). The processed data including peak area, peak area ratio, retention time, and concentration were exported into Microsoft Excel to create an appropriate file for input in the R software [20]. The normality of the variables was checked using the Kolmogorov-Smirnov test and visually checked using quantile-quantile plots, and the equality of variances was examined using Welch's F-test. Outliers were identified using Grubbs's test. To compare data between the control and multiple sclerosis groups, box plots with SDs were generated. Comparisons between the two groups were conducted using an independent samples $t$-test or two-sample Wilcoxon test in R. A $p$-value of $<0.05$ was considered statistically significant.

\section{Results and discussion}

\subsection{Optimization of sample preparation}

The analysis of small molecules using LC-MS usually requires cleanup procedures to eliminate the worst interfering compounds and to concentrate the sample if the analyte is present at extremely low concentrations. Biological samples contain proteins that adversely affect the analysis of small molecules. To improve sensitivity, proteins in samples had to be eliminated. In our preliminary experiments, four different precipitation solvents were compared with their $3 \times$ and $5 \times$ volumes in human serum samples [i.e., $\mathrm{MeOH}, \mathrm{ACN}$, acetone- $\mathrm{MeOH}(3: 7, \mathrm{v} / \mathrm{v})$, and acetone-MeOH $(1: 1, v / v)]$ and two different solvents in CSF samples (i.e., $\mathrm{MeOH}$ and ACN) to monitor the recovery of each TRP metabolite. 3-HANA could not be detected using $\mathrm{MeOH}$ (data not shown). The best result was achieved using $3 \times$ volume of $\mathrm{ACN}$ to precipitate the majority of proteins in CSF samples and $3 \times$ volume of acetone-MeOH (1:1, $\mathrm{v} / \mathrm{v}$ ) for serum samples (see Chapter 3.3.3).

In a complex quantitative bioanalytical method, the use of SIL-IS can help control the variability of the method. Because it is processed along with the analyte, SIL-IS should both help correct for variability in sample preparation during extraction and chemical derivatization and compensate for variability in MS detection. In the present study, 11 deuterated equivalents of TRP metabolites were added at the beginning of analysis to control every step of the analytical procedure. Unfortunately, the deuterated form of 
Table 2

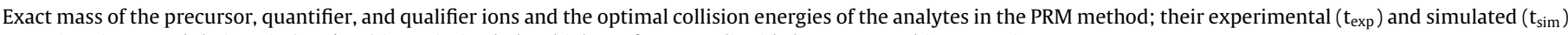
retention times, and their ratio $\left(t_{\exp } / t_{\text {sim }}\right)$ in optimized ultra high-performance liquid chromatographic separation.

\begin{tabular}{|c|c|c|c|c|c|c|c|}
\hline Analyte & Precursor ion $[\mathrm{M}+\mathrm{H}]^{+}$ & Quantifier ion $[\mathrm{M}+\mathrm{H}]^{+}$ & Qualifier ion $[\mathrm{M}+\mathrm{H}]^{+}$ & $\mathrm{CE}(\mathrm{eV})$ & $\mathrm{t}_{\exp }(\min )$ & $\mathrm{t}_{\mathrm{sim}}(\min )$ & $\mathrm{t}_{\exp } / \mathrm{t}_{\mathrm{sim}}$ \\
\hline SERO & 177.1022 & 115.0548 & 132.0810 & 32 & 1.53 & 1.50 & 1.02 \\
\hline d4-SERO & 181.1273 & 119.0704 & 136.1060 & 32 & 1.53 & 1.50 & 1.02 \\
\hline KYN & 209.0919 & 94.0660 & 146.0603 & 14 & 1.61 & 1.59 & 1.01 \\
\hline d4-KYN & 213.1169 & 98.0912 & 150.0856 & 14 & 1.61 & 1.59 & 1.01 \\
\hline 3-HANA & 154.0499 & 136.0393 & 108.0453 & 10 & 2.26 & 2.24 & 1.01 \\
\hline d3-3-HANA & 157.0688 & 139.0582 & 111.0812 & 10 & 2.26 & 2.24 & 1.01 \\
\hline TRP & 205.0973 & 118.0652 & 188.0706 & 18 & 2.42 & 2.38 & 1.01 \\
\hline d5-TRP & 210.1285 & 123.0968 & 193.1018 & 18 & 2.42 & 2.38 & 1.01 \\
\hline 5-HIAA & 192.0659 & 146.0600 & 117.0570 & 18 & 2.69 & 2.65 & 1.01 \\
\hline d5-5-HIAA & 197.0972 & 150.0852 & 122.0685 & 18 & 2.69 & 2.65 & 1.01 \\
\hline ANA & 138.0547 & 92.0503 & 120.0449 & 20 & 3.06 & 3.03 & 1.01 \\
\hline KYNA & 190.0502 & 116.0503 & 162.0552 & 38 & 3.54 & 3.55 & 1.00 \\
\hline d5-KYNA & 195.0806 & 121.0816 & 167.0867 & 38 & 3.54 & 3.55 & 1.00 \\
\hline $\mathrm{XA}$ & 206.0450 & 178.0508 & 132.0443 & 21 & 3.90 & 3.91 & 1.00 \\
\hline d4-XA & 210.0695 & 182.0761 & 136.0691 & 21 & 3.90 & 3.91 & 1.00 \\
\hline 3-HK & 281.1496 & 152.0709 & 110.0606 & 15 & 3.99 & 4.03 & 0.99 \\
\hline d3-3-HK & 284.1684 & 155.0897 & 113.1082 & 15 & 3.99 & 4.03 & 0.99 \\
\hline MELA & 233.1284 & 130.0654 & 115.0546 & 54 & 4.11 & 4.16 & 0.99 \\
\hline d4-MELA & 237.1536 & 134.0907 & 119.0801 & 54 & 4.11 & 4.16 & 0.99 \\
\hline PICA & 180.1019 & 96.0453 & 124.0396 & 28 & 4.38 & 4.43 & 0.99 \\
\hline d4-PICA & 184.1270 & 100.0702 & 128.0647 & 28 & 4.38 & 4.43 & 0.99 \\
\hline QUIN & 280.1543 & 96.0453 & 124.0396 & 37 & 4.63 & 4.61 & 1.00 \\
\hline d3-QUIN & 283.1732 & 99.0641 & 128.0647 & 37 & 4.63 & 4.61 & 1.00 \\
\hline
\end{tabular}

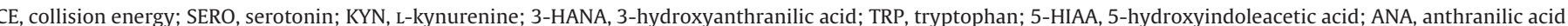
KYNA, kynurenic acid; XA, xanthurenic acid; 3-HK, 3-hydroxykynurenine; MELA, melatonin; PICA, picolinic acid; QUIN, quinolinic acid.

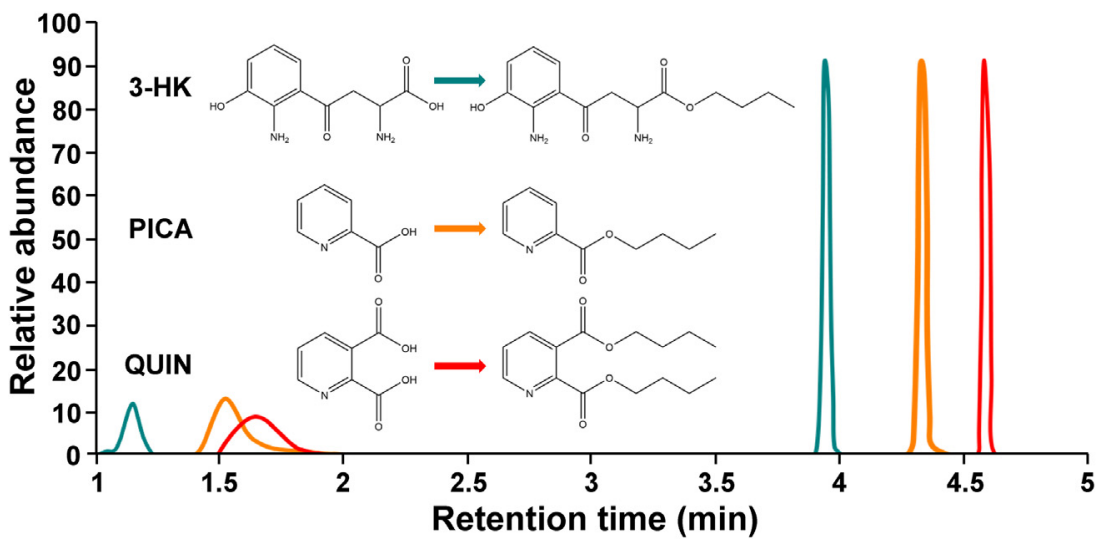

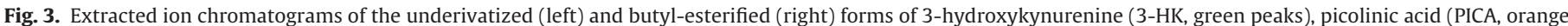
peaks), and quinolinic acid (QUIN, red peaks).

ANA was unavailable; therefore, for its quantitative estimation, d5-KYNA, which has a similar chromatographic elution time, was used.

Some TRP metabolites (3-HK, PICA, and QUIN) have unfavorable chromatographic properties, making their quantitative LC-MS estimation unreliable. The chromatographic properties of analytes can be modified via derivatization. Changes in the structure of an analyte usually affect its physical and chemical properties (e.g., polarity, solubility, stability, and ionization efficiency in mass spectrometry), which can alter the separation characteristics of the analyte (e.g., improved peak shape, elution time, peak symmetry, efficiency, and plate count) [21]. If these changes are favorable, they might lead to improvement in the separation of the analyte. Regarding precolumn derivatization, the selected reaction must be quantitative or, at least, reproducible and free of byproducts.

At the same time, chemical modification may serve to minimize matrix interference by moving the compound to a position on the chromatogram where interference with the matrix component is minimal.

Except SERO and MELA, TRP and its metabolites have carboxyl $\operatorname{group}(\mathrm{s})$. The most frequently used approach for derivatizing car- boxylic acids is esterification with a short-chain aliphatic alcohol in the presence of an acid as a catalyst [22]. Although this established esterification method (Fischer esterification) leads to equilibrium, the reaction can be shifted toward the products by applying excess amount of alcohol. Esterification of TRP and its metabolites was performed using $\mathrm{MeOH}$, ethanol, $n$-propanol, or $n$-butanol. Because they have the longest hydrophobic aliphatic chains, the butylated products exhibited the highest retention when reversed-phase columns were used, and there was no coelution of the esterified and nonderivatized components (data not shown). Butyl ester formation changed the polarity of the molecules, resulting in the formation of well-retained peaks with excellent peak shapes in cases of 3-HK, PICA, and QUIN (Fig. 3). Esterification of analytes with the mixture of $n$-butanol and acetyl chloride is a commonly used procedure to derivatize amino acids for newborn amino acid screening via mass spectrometry [22].

The derivatization method was optimized by assessing the effect of reaction time $(0,20,30,40,50$, and $60 \mathrm{~min} ; \mathrm{n}=3$; Fig. 4$)$, and the maximum conversion of carboxyl group(s) to esters was achieved after 60 -min reaction. Although esterification was not complete after 60 min for all components (74\%-95\%), the method could be 
Table 3

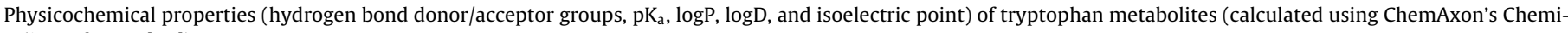
calize software [24])

\begin{tabular}{|c|c|c|c|c|c|c|c|c|c|c|c|}
\hline Analyte & Molecular mass $(\mathrm{g} / \mathrm{mol})$ & $\mathrm{pK}_{\mathrm{a} 1}$ & $\mathrm{pK}_{\mathrm{a} 2}$ & $\mathrm{pK}_{\mathrm{a} 3}$ & $\mathrm{pI}$ & $\log \mathrm{P}$ & $\log \mathrm{D}(\mathrm{pH} 3)$ & $\log \mathrm{D}(\mathrm{pH} 6)$ & $\log \mathrm{D}(\mathrm{pH} 9)$ & $\begin{array}{l}\text { Hydrogen bond } \\
\text { donor count }\end{array}$ & $\begin{array}{l}\text { Hydrogen bond } \\
\text { acceptor count }\end{array}$ \\
\hline SERO & 212.68 & 9.31 & 10.00 & & 9.78 & 0.48 & -1.85 & -1.76 & 0.32 & 3 & 2 \\
\hline KYN & 306.29 & 1.19 & 8.96 & & 6.11 & -1.91 & -2.35 & -1.91 & -2.20 & 3 & 5 \\
\hline 3-HANA & 153.14 & 1.94 & 10.37 & 4.82 & 3.03 & 1.15 & 0.75 & -0.42 & -2.36 & 3 & 4 \\
\hline TRP & 204.23 & 2.54 & 9.40 & & 5.97 & -1.09 & -1.19 & -1.09 & -1.22 & 3 & 3 \\
\hline 5-HIAA & 191.18 & 4.22 & 9.56 & & - & 1.41 & 3.80 & -0.38 & -2.16 & 3 & 3 \\
\hline ANA & 137.14 & 4.89 & 1.95 & & 3.34 & 1.45 & 1.27 & 0.17 & -2.00 & 2 & 3 \\
\hline KYNA & 189.17 & 2.47 & & & 2.31 & 1.87 & 2.40 & -0.47 & -1.68 & 2 & 4 \\
\hline $\mathrm{XA}$ & 205.17 & 2.17 & 9.06 & 14.22 & 3.25 & -0.17 & -0.17 & -0.65 & -2.22 & 3 & 5 \\
\hline 3-HK & 224.21 & 0.99 & 9.86 & 3.378 .90 & 6.11 & -2.21 & -2.73 & -2.21 & -2.57 & 4 & 6 \\
\hline MELA & 232.28 & -1.57 & 15.90 & & 7.08 & 1.15 & 1.15 & 1.15 & 1.15 & 2 & 2 \\
\hline PICA & 123.11 & 1.00 & 5.52 & & 3.04 & -0.65 & -0.65 & -1.25 & -2.71 & 1 & 3 \\
\hline QUIN & 167.12 & 0.31 & 4.16 & 6.67 & 2.27 & -1.2 & -1.23 & -3.11 & -6.39 & 2 & 5 \\
\hline
\end{tabular}

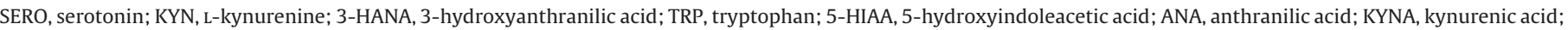
XA, xanthurenic acid; 3-HK, 3-hydroxykynurenine; MELA, melatonin; PICA, picolinic acid; QUIN, quinolinic acid.

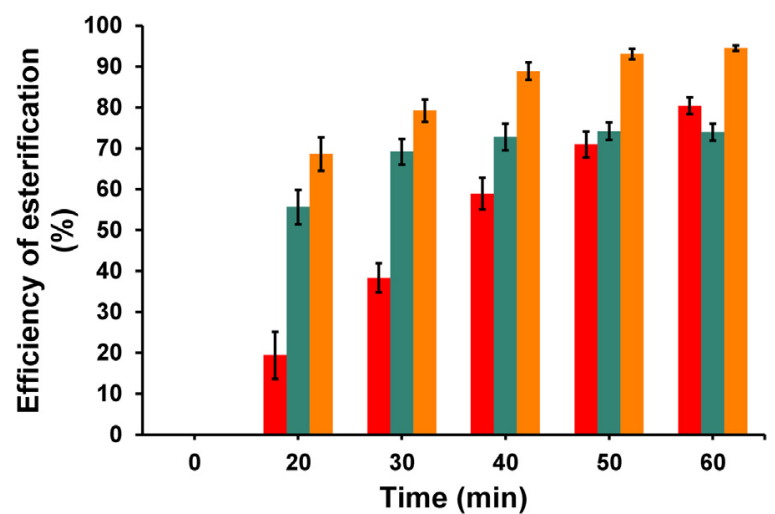

Fig. 4. Efficiency of esterification in percentages at different reaction times. Quinolinic acid, red; 3-hydroxykynurenine, green; picolinic acid, orange.

reliably used because of the presence of SIL-IS, which has nearly identical chemical and physical properties as the target analyte. Although the absolute response may be affected, the analyte/IS peak area ratio should be unaffected and the method should be accurate, precise, and rugged.

During sample preparation, all carboxyl group-containing compounds were esterified in $50 \%$ of the samples and remixed with the untreated fraction to analyze butylated 3-HK, PICA, and QUIN in the same chromatographic run with SERO, MELA, and the non-esterified carboxyl group-containing TRP and its remaining metabolites.

\subsection{Development and optimization of the UHPLC method}

LC-MS is one of the most prominent analytical techniques because of its inherent selectivity and sensitivity. Most currently used HPLC/UHPLC separation techniques are performed using reversed-phase chromatography (RPC). RPC has become the standard technique for analyzing a wide range of small (even large) compounds ranging from neutral polar and nonpolar solutes to acidic, basic, and amphoteric compounds. Stationary phases used in RPC typically comprise varying lengths of hydrocarbons such as $\mathrm{C} 18, \mathrm{C} 8$, and $\mathrm{C} 4$, for which analyte retention is mainly driven by hydrophobic and van der Waals interactions. As the mobile phase, a mixture of water with a miscible, polar organic solvent such as ACN and $\mathrm{MeOH}$ is used, usually supplemented with different additives.

TRP and some of its metabolites are rather hydrophobic because of the indole or phenyl ring; however, the attached pyridine ring and hydroxy, amine, or carboxy groups increase their hydrophilicity while providing ionic properties to molecules. Developing an
LC-MS method for the simultaneous quantitative estimation of these types of target compounds is extremely challenging because of the great diversity in their physicochemical properties (e.g., $\mathrm{pK}_{\mathrm{a}}$, isoelectric point, hydrophobicity, number of hydrogen bond donor/acceptor groups, and solubility; Table 3).

The first stage of chromatographic method development is identifying the most promising column chemistry, organic modifier, and $\mathrm{pH}$ of the mobile phase for analyte separation. To scout the most appropriate column and organic solvent, we performed two initial experiments using a number of columns with elution using a MS-compatible generic gradient (see Section 2.5.1). The "best" column and organic modifier were selected via visual comparison of the resultant chromatograms, taking into account the overall peak shapes, retention of highly polar compounds, and baseline separation of analytes in the shortest time. Then, DryLab ${ }^{\circledR} 4$ was used to optimize separation on the selected column.

An octadecylsilane column is the first choice for separating TRP and its metabolites; therefore, we tested seven C18 columns. Separation was also performed on reversed-phase columns containing other hydrocarbon groups ( $\mathrm{C} 8, \mathrm{C} 12$, and phenyl-hexyl). Because of the different characteristics of the tested stationary phases, they exhibited distinct retention and elution profiles. PICA was the first eluting analyte on all columns, but satisfactory retention was observed only using the BEH C18 and Luna Omega Polar columns. The early eluting QUIN could not be detected using the Kinetex C18 and Cortecs $\mathrm{C} 18$ columns, whereas it appeared as a wide peak when the BEH C18 and Luna C18 columns were used. Its elution profile was unacceptably wide on the other columns. 3-HK exhibited low retention, and it sometimes eluted as double peaks (Luna C18, BEH C18, Polar C18, and Cortecs C18 columns). The peak of SERO had fronting and tailing on the Kinetex C18, Luna C18, BEH C18, and Cortecs $\mathrm{C} 18$ columns. The peak shape of XA was unacceptable on the Luna C18, Kinetex C18, Cortecs C18, and phenyl-hexyl columns. TRP, 5-HIAA, ANA, KYNA, and XA grouped at the second part of chromatograms in 0.6-1.3-min windows usually as unseparated peaks. Stationary phases with shorter alkyl chains proved to be even less retentive for these analytes; therefore, we had to search for other types of reversed-phase columns.

Retention times were shorter and peaks were somewhat sharper using ACN as an organic modifier; however, no important selectivity or sensitivity differences were observed.

Fluorinated stationary phases, particularly those involving PFP moieties, have become popular alternatives to the traditional alkyl phases because of the differences in selectivity and retention that they provide. PFP phases use multiple retention mechanisms to separate small, highly polar aromatic compounds [23], and they appear to be ideal choices for analyzing TRP metabolites. The elec- 
tronegative fluorine atoms produce an electron-deficient phenyl ring, which permits the PFP phase to act as a Lewis acid or an electron acceptor. $\pi-\pi$ interactions can occur with solutes rich in electrons (Lewis bases) such as amino and hydroxyl groups. Carbon-fluorine bonds are extremely polar, thus enabling analytes to also be retained by dipole-dipole interactions, H-bonding, and sometimes ionic interactions, resulting in increased analyte retention. In the presence of a rigid aromatic ring, the solute shape can also dictate selectivity. The predominance of each retention mechanism is influenced by the physicochemical properties and structure of analytes and the chromatographic conditions used.

Core-shell Kinetex F5 $(2.1 \times 150 \mathrm{~mm}, 2.6 \mu \mathrm{m})$ and two Kinetex PFP $(2.1 \times 100 \mathrm{~mm}, 2.6 \mu \mathrm{m}$; Phenomenex $)$ columns with different lot numbers were tested using the abovementioned generic gradients. Similarly, as observed for the C18 stationary phases, PICA, QUIN, and 3-HK eluted in wide, skewed peaks from every fluorinated phase. Despite the different structures (distinct functional groups on benzene, pyridine, or indole rings), the $\mathrm{pK}_{\mathrm{a}}$ values and $\mathrm{H}$-bonding capabilities of KYN-SERO and 5-HIAA-KYNA-XA pairs could not be resolved on the Kinetex F5 column, as observed for 3-HANA-KYN and XA-TRP pairs on the Kinetex PFP column (from newer series). The older Kinetex PFP column could separate the abovementioned analyte pairs using $\mathrm{MeOH}$ as an organic modifier, and it generally produced a "good-looking" chromatogram (except PICA, QUIN, and 3-HK). Choosing ACN instead of MeOH resulted in differences in selectivity; however, 3-HANA and TRP were coeluted, followed by deteriorated quantification of 3-HANA because the concentration of TRP in serum and CSF are at least three orders of magnitude higher than that of 3-HANA. Similarly, ANA, KYNA, and XA could not be resolved using ACN.

Because of the different ionizable functional groups of TRP and its metabolites, the $\mathrm{pH}$ of the mobile phase could influence the ionization state of these compounds. However, because 10 of our analytes possess carboxyl group(s), their ionization was suppressed at $\mathrm{pH}<3$, resulting in more hydrophobic species. The majority of these metabolites are zwitterionic molecules, and 7 of them have isoelectric points below 6.0, indicating that the carboxyl group is in the anionic form around $\mathrm{pH}$ 6. At higher $\mathrm{pH}$ values, more of these compounds have additional deprotonized functional groups, resulting in anionic species. Usually, detection of anionic analytes via mass spectrometry in the negative mode results in lower sensitivity. Moreover, almost all of our tested columns are reported by their respective manufacturers to have poor stability above $\mathrm{pH} 8$.

The same conclusion can be drawn from the calculated logP and $\log D$ values presented in Table 3 [24]. In reversed-phase HPLC, retention time is closely related to the hydrophobicity of analytes, which can be estimated using $\log \mathrm{P}$ (octanol/water) values. When an analyte is dissociated in the mobile phase, $\log \mathrm{D}$ (distribution coefficient, which reflects the contribution of all ionic species to the total hydrophobicity of an analyte) is often used instead of $\log$ P. Increasing $\mathrm{pH}$ resulted in lower $\log \mathrm{D}$ values for almost all tested analytes, which again resulted in lower retention time.

Based on the results of column- and organic modifier-scouting experiments and the theoretical consideration of the $\mathrm{pH}$ of the mobile phase, separation of TRP and its metabolites was performed on a Kinetex PFP column under acidic conditions using $0.1 \%(\mathrm{v} / \mathrm{v})$ aqueous FA ( $\mathrm{pH} 2.7$ ) and $\mathrm{MeOH}$ as an organic modifier.

Derivatization of the carboxyl group(s) of TRP and 9 of its metabolites via esterification with four carbon atoms containing $n$-butanol increased the hydrophobicity of analytes, resulting in higher retention on reversed-phase chromatographic columns. The peak shape of the most polar compounds 3-HK, PICA, and QUIN was optimal after butylation (Fig. 3), and their retention increased significantly. QUIN has two carboxyl groups, and their esterification made QUIN the most hydrophobic TRP metabolite. Using a generic gradient (see Section 2.5.1), the least retentive esterified
3-HK eluted immediately before the most retentive nonesterified metabolite MELA. Esterified 5-HIAA and KYN coeluted, whereas 5 butylated metabolites (TRP, KYNA, 3-HANA, XA, and ANA) grouped between esterified PICA and QUIN without disturbing the determination of other analytes.

Considering the chromatographic behavior of esterified and nonesterified TRP and its metabolites, 7 compounds were selected for their quantitative estimation in the nonderivatized forms, whereas 3 metabolites were quantitatively estimated in their butylated forms. SERO and MELA were also analyzed (nonesterifiable), but their in vivo concentrations proved to be lower than their LOD in the case of CSF samples. However, SERO could be quantified in serum samples.

Apart from the type and physical characteristics of the stationary phase, nature of organic modifier, and composition and $\mathrm{pH}$ of the mobile phase, other chromatographic parameters (e.g., length and profile of the gradient, $T$, ionic strength of the mobile phase, initial and final organic concentrations of eluents, and column dimensions) can also influence analyte separation. Improving the analytical performance testing of these parameters would be tedious and time-consuming, but not cost-effective or "green." However, computer-assisted HPLC method development/optimization software can help define the appropriate conditions for robust, precise, and reproducible analysis and can simultaneously save resources. DryLab ${ }^{\circledR} 4$ is one such software that helps the chromatographer develop better and more reliable HPLC/UHPLC methods in a shorter time. This can be achieved by in silico modeling of retention and resolution based on a limited number (2-12) of initial experiments [13].

To optimize separation of TRP and its 11 metabolites on a Kinetex PFP column using $\mathrm{MeOH}$ as an organic modifier, we performed four initial linear gradient chromatographic runs to test the effects of gradient steepness $/ \mathrm{t}_{\mathrm{G}}\left(\mathrm{t}_{\mathrm{G} 1}=5\right.$ and $\left.\mathrm{t}_{\mathrm{G} 2}=15 \mathrm{~min}\right)$ and $\mathrm{T}\left(\mathrm{T}_{1}=25^{\circ} \mathrm{C}\right.$ and $\mathrm{T}_{2}=50^{\circ} \mathrm{C}$ ) on retention time and resolution. Based on these chromatograms, the software simulated chromatograms and created a 2D color-coded resolution map, plotting critical resolution as a function of $t_{G}$ and $T$. Red regions on this plot represent the optimal chromatographic conditions with a resolution of $>1.3$. We selected a working point $\left(\mathrm{t}_{\mathrm{G} 1}=5 \mathrm{~min}, \mathrm{~T}=25^{\circ} \mathrm{C}\right)$, and using the gradient editor, we simulated the resolution of the two most problematic analyte pairs: SERO-KYN and 3-HK-XA (Fig. 5). The best separation was achieved using a gradient with four linear segments: $0-1 \mathrm{~min}$, $10 \%-30 \% \mathrm{~B} ; 1-3 \mathrm{~min}, 30 \%-50 \% \mathrm{~B} ; 3-3.5 \mathrm{~min}, 50 \%-90 \% \mathrm{~B}$; and 3.5-5 min, 90 \% B (Fig. 6). Retention time prediction was experimentally verified using the selected parameters. The experimental $\left(t_{\text {exp }}\right)$ and simulated $\left(t_{\text {sim }}\right)$ retention times for each analyte, together with their ratio $\left(r=t_{\exp } / t_{\text {sim }}\right)$, are summarized in Table 2 . All ratios ranged from 0.99 to 1.02 , indicating an excellent match between the simulated and actual separations.

DryLab $^{\circledR} 4$ combines over 30 years of HPLC expertise with the latest software technologies and enables chromatographers involved in pharmaceutical and chemical industries, environmental protection, and research industries to easily create fast, robust, high-quality methods [21]. To the best of our knowledge, this is the first report on the use of DryLab ${ }^{\circledR} 4$ to optimize LC/MS analysis of endogenous multianalyte-containing biological samples [25].

\subsection{Method validation}

\subsubsection{Linearity, $L O D$, and $L O Q$}

The LOD, LOQ, retention time, and linearity of the calibration curves with $\mathrm{r}^{2}$ values are shown in Supplementary Table 1 for CSF and Supplementary Table 2 for serum. For all analytes, $\mathrm{r}^{2}$ exceeded 0.99. LOD was less than $7.80 \mathrm{nM}$, and in most cases, LOQ was lower than $8.26 \mathrm{nM}$ for all metabolites, except in SERO and QUIN (23.65 and $20.59 \mathrm{nM}$, respectively). 


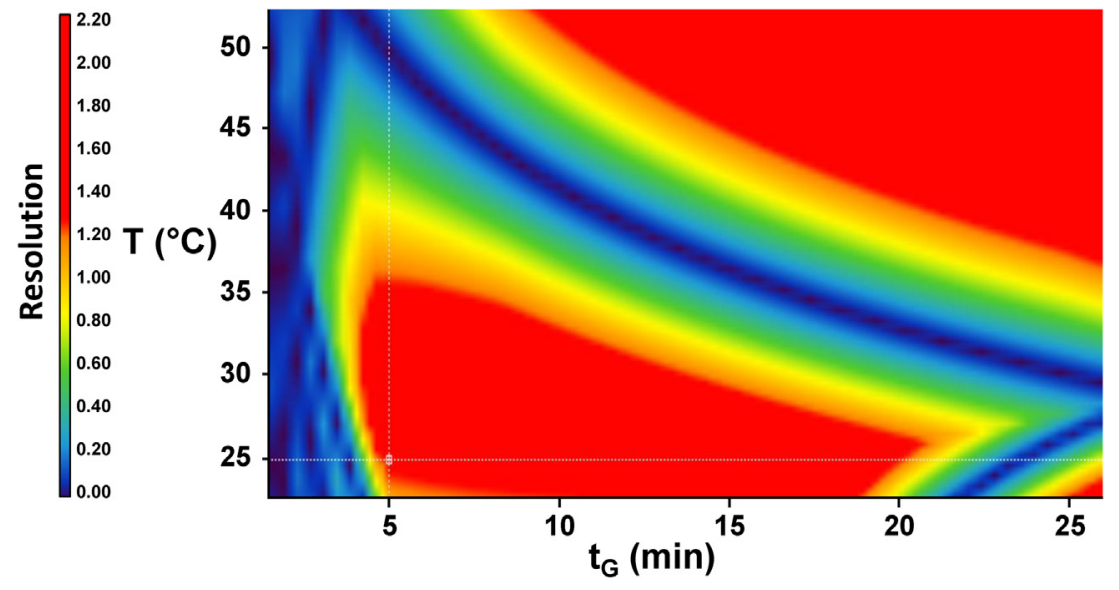

Fig. 5. DryLab ${ }^{\circledR} 4$ resolution map with working point $\left(t_{G}=5 \mathrm{~min}, \mathrm{~T}=25^{\circ} \mathrm{C}\right)$.

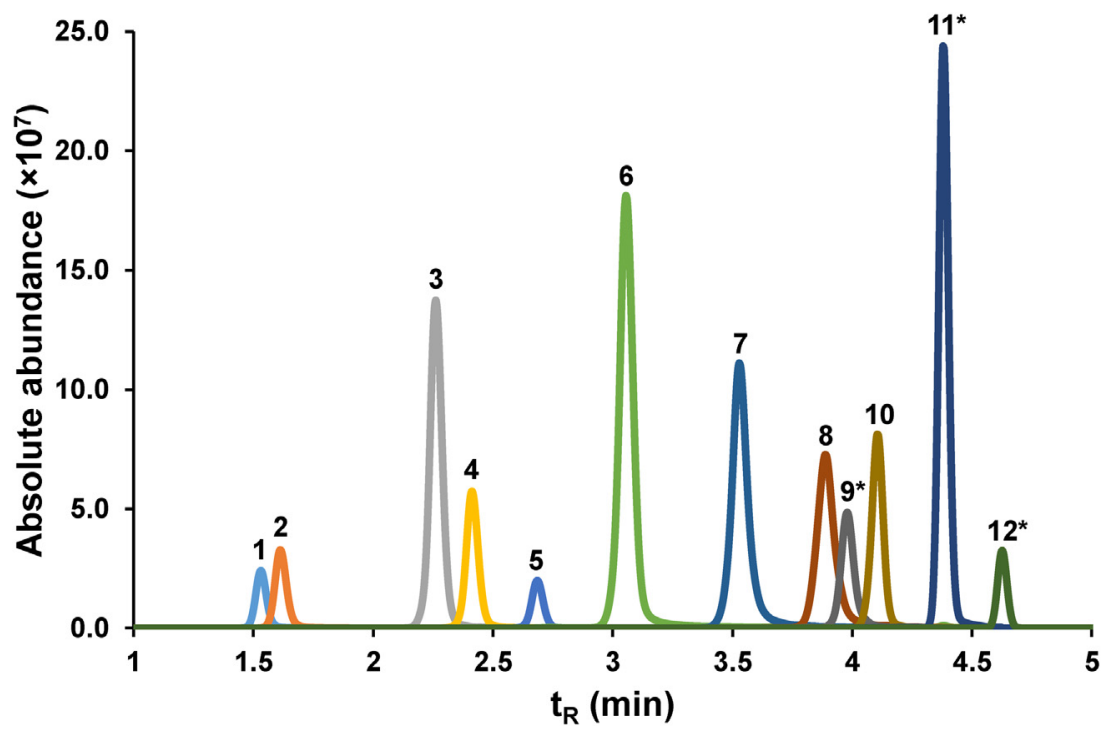

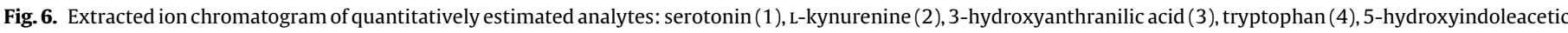

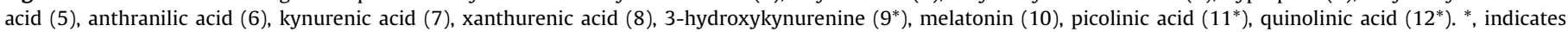
derivatized analytes.

\subsubsection{Precision and accuracy}

The intraday and interday precisions (RSD) of the method for the two matrices were obtained by analyzing five replicates of the three QC levels on 3 consecutive days (Supplementary Tables 3-6). According to the results, the developed method was found to have a reliable precision.

The accuracy ranged from 86.7-112.0\% of intraday and interday measurements (Supplementary Tables 3 and 4) in aCSF and from 89.1-107.7 \% in the "blank" serum (Supplementary Tables 5 and 6), which are in line with recommended data $[17,18]$.

\subsubsection{Recovery}

To measure analyte concentrations, a simple protein precipitation method was selected instead of liquid-liquid or solid-phase extraction. In general, protein precipitation is easier, more rapid, and reasonable than the abovementioned methods. The process first was conducted using ACN and $\mathrm{MeOH}$ for aCSF and with supplementation with acetone- $\mathrm{MeOH}(3: 7, \mathrm{v} / \mathrm{v})$ and acetone-MeOH $(1: 1, v / v)$ for the "blank" serum. Finally, for protein precipitation, ACN was used for aCSF and acetone-MeOH $(1: 1, v / v)$ for the "blank" serum. Analyte recoveries were determined at three different concentrations to prove that the recovery of each analyte was concentration independent, reproducible, and consistent.

Analyte recoveries ranged from 93.8-105.3 \% for aCSF (Supplementary Table 7) and from 84.7-109.4\% for the "blank" serum (Supplementary Table 8 ). Our values are within the range recommended by the ICH and FDA guidelines $[17,18]$.

\subsection{TRP metabolite profile changes in multiple sclerosis}

In most cases, the quantitative estimation of many analytes with different polarities is challenging. Hence, derivatization is commonly used to improve the selectivity and sensitivity of the method because it changes the chemical properties of compounds and increases the mass of analytes, which provide an excellent strategy for avoiding the interference of matrix components [26]. Therefore, to achieve successful simultaneous quantitation of the 12 analytes, a derivatization step was included to improve the peak shape and retention of 3-HK, PICA, and QUIN. Although the concentrations of both SERO and MELA were below their LOD in CSF and that of MELA was also below its LOD in serum, the method was validated according to the ICH and FDA guidelines [17,18]. Applicability of the method was proven by quantifying the metabolites of 

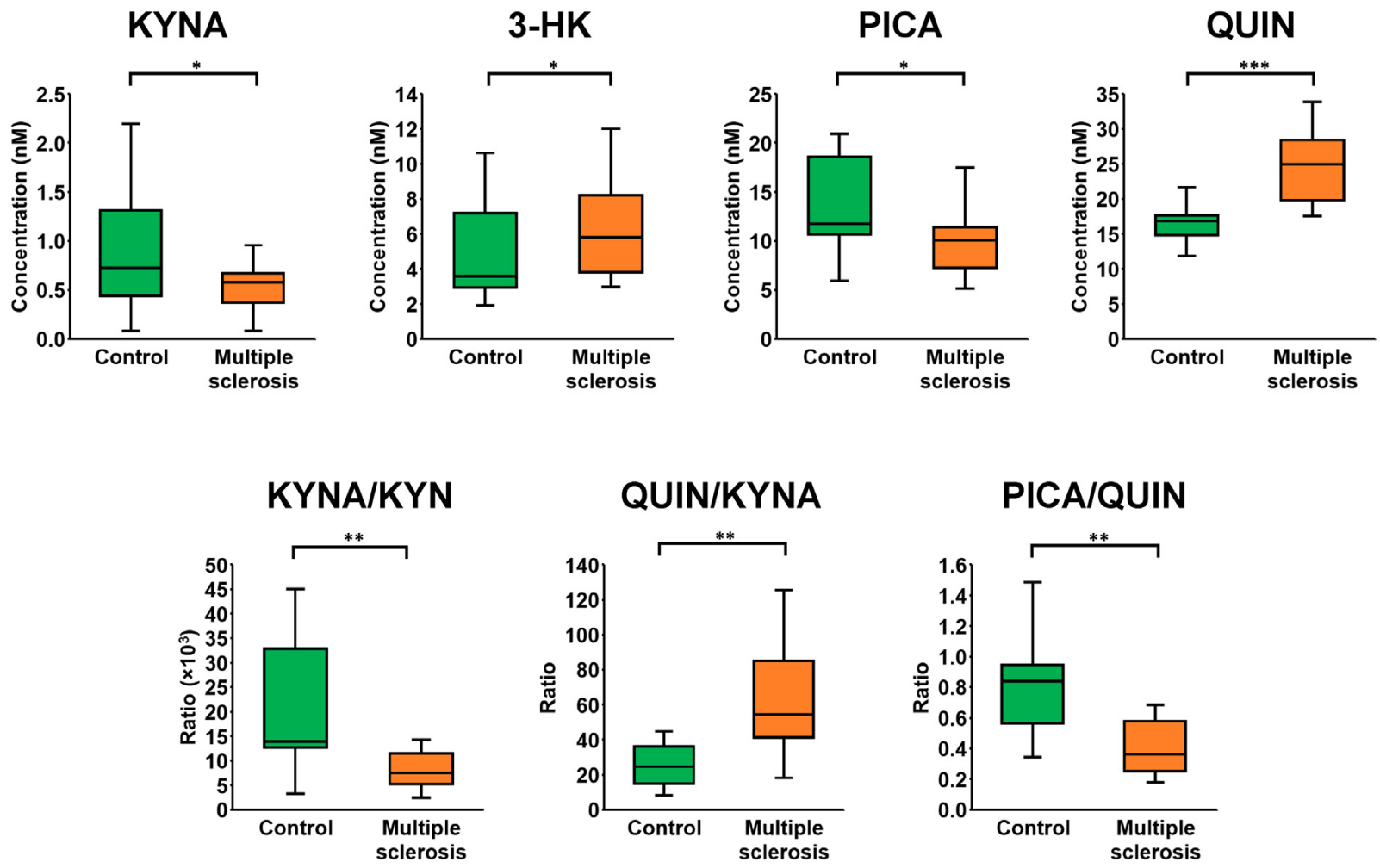

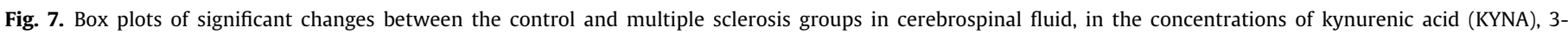

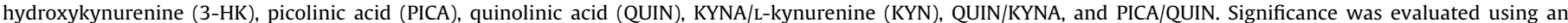
independent samples $t$-test or a two-sample Wilcoxon test after the F-test: ${ }^{*} \mathrm{p}<0.05 ;{ }^{* *} \mathrm{p}<0.01 ;{ }^{* * *} \mathrm{p}<0.001$.

Table 4

Concentrations of TRP and its metabolites in human cerebrospinal fluid and serum in the control and multiple sclerosis groups.

\begin{tabular}{|c|c|c|c|c|c|c|}
\hline & CSF & & & Serum & & \\
\hline Analyte & $\begin{array}{l}\text { Control group } \\
\text { Concentration (nM) }\end{array}$ & Multiple sclerosis group & $p$-value & $\begin{array}{l}\text { Control group } \\
\text { Concentration (nM) }\end{array}$ & Multiple sclerosis group & $p$-value \\
\hline SERO & $<$ LOD & $<$ LOD & - & $919.9 \pm 451.1$ & $725.9 \pm 365.4$ & 0.1761 \\
\hline KYN & $49.3 \pm 16.6$ & $60.4 \pm 21.2$ & $0.1691^{\mathrm{a}}$ & $2397 \pm 664.4$ & $2365 \pm 714.9$ & 0.8949 \\
\hline 3-HANA & $0.7 \pm 0.7$ & $1.1 \pm 0.8$ & $0.1533^{a}$ & $58.0 \pm 20.6$ & $55.3 \pm 16.8$ & 0.6853 \\
\hline TRP & $1847 \pm 305.6$ & $1781 \pm 367.3$ & $0.9277^{a}$ & $52043 \pm 11057$ & $50948 \pm 9325$ & 0.4089 \\
\hline 5-HIAA & $101.0 \pm 36.7$ & $85.8 \pm 31.4$ & 0.3487 & $66.0 \pm 21.3$ & $50.7 \pm 11.3$ & 0.0248 \\
\hline ANA & $7.0 \pm 3.9$ & $6.3 \pm 3.0$ & $0.7957^{a}$ & $16.2 \pm 6.3$ & $15.9 \pm 5.0$ & 0.9047 \\
\hline KYNA & $0.9 \pm 0.6$ & $0.5 \pm 0.2$ & 0.0469 & $32.2 \pm 10.4$ & $27.2 \pm 9.5$ & 0.1692 \\
\hline $\mathrm{XA}$ & $0.037 \pm 0.034$ & $0.053 \pm 0.062$ & $0.8534^{\mathrm{a}}$ & $13.7 \pm 9.0$ & $10.6 \pm 5.0$ & $0.5981^{\mathrm{a}}$ \\
\hline 3-HK & $4.7 \pm 2.8$ & $6.4 \pm 2.6$ & $\mathbf{0 . 0 3 4 6}^{\mathrm{a}}$ & $92.0 \pm 46.3$ & $89.8 \pm 27.9$ & 0.8724 \\
\hline MELA & $<$ LOD & $<$ LOD & - & $<$ LOD & $<$ LOD & - \\
\hline PICA & $13.4 \pm 4.6$ & $10.0 \pm 3.4$ & 0.0224 & $35.4 \pm 8.4$ & $31.6 \pm 9.2$ & 0.2586 \\
\hline QUIN & $16.4 \pm 2.8$ & $24.7 \pm 5.1$ & 0.0001 & $100.5 \pm 45.9$ & $158.0 \pm 73.5$ & 0.0302 \\
\hline QUIN/KYNA & $36.2 \pm 37.1$ & $73.2 \pm 57.8$ & $\mathbf{0 . 0 0 1 5}^{\mathrm{a}}$ & $3.7 \pm 2.0$ & $6.5 \pm 2.9$ & 0.0183 \\
\hline $\mathrm{KYN} / \mathrm{TRP}\left(\times 10^{3}\right)$ & $28.2 \pm 11.1$ & $35.1 \pm 14.5$ & 0.1575 & $48.2 \pm 15.8$ & $46.8 \pm 13.5$ & $0.9855^{\mathrm{a}}$ \\
\hline PICA/QUIN & $0.8 \pm 0.4$ & $0.4 \pm 0.2$ & 0.0065 & $0.4 \pm 0.3$ & $0.2 \pm 0.2$ & 0.0476 \\
\hline KYNA/KYN $\left(\times 10^{3}\right)$ & $18.5 \pm 12.3$ & $8.9 \pm 5.0$ & $0.0041^{a}$ & $14.2 \pm 5.6$ & $11.5 \pm 3.3$ & $0.0832^{\mathrm{a}}$ \\
\hline
\end{tabular}

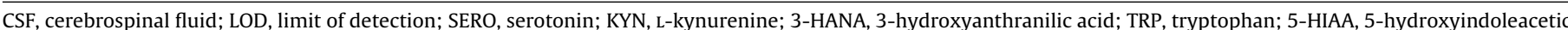
acid; ANA, anthranilic acid; KYNA, kynurenic acid; XA, xanthurenic acid; 3-HK, 3-hydroxykynurenine; MELA, melatonin; PICA, picolinic acid; QUIN, quinolinic acid.

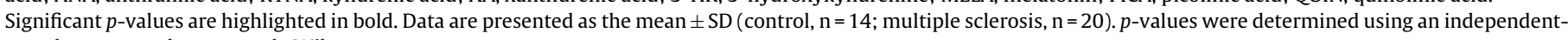
samples $t$-test and two-sample Wilcoxon test.

a Two-sample Wilcoxon test.

interest from both human CSF and serum in a short study, in which metabolite concentrations were compared between the multiple sclerosis and control groups.

Investigation of the molecular background of neurodegenerative diseases such as multiple sclerosis requires intensive research, and some analytes derived from TRP have been studied comprehensively $[1,2,5,8]$. The need to measure all important metabolites in a single run is necessary considering the efficiency, financial, and time-saving aspects of a method, whereas robustness ensures the applicability of the same method on different biological matrices, which is crucial for better comprehension of the actual pathomechanisms of diseases.

In a recent study [8] of TRP metabolites, changes in KYNA, PICA, QUIN, and 3-HK concentrations were compared between patients with multiple sclerosis and controls. Our findings are in line with literature data, and QUIN-induced excitotoxic effects can be counterbalanced by KYNA. In the context of multiple sclerosis, QUIN concentration increased dramatically in both CSF and serum, whereas KYNA concentration slightly decreased (Table 4 and Figs. 7 and 8), resulting in a significantly higher QUIN/KYNA 

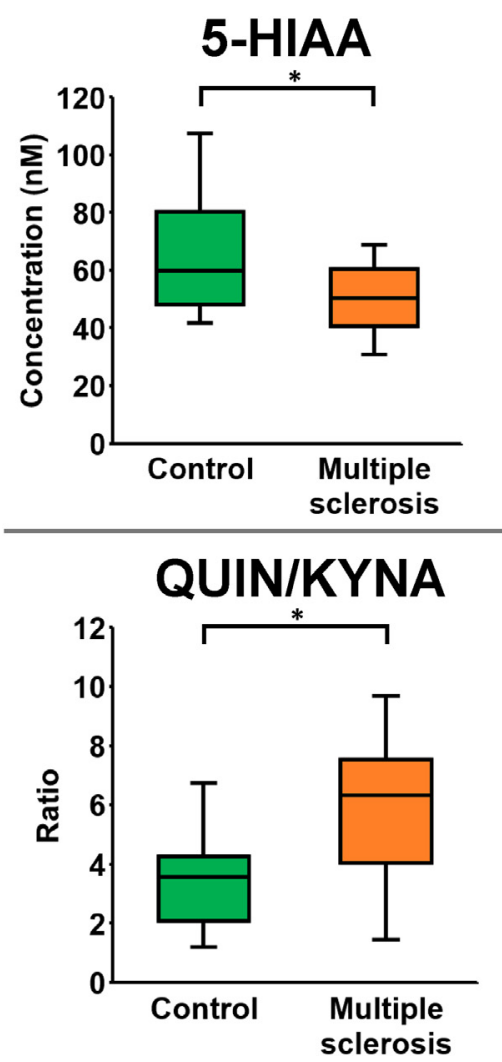
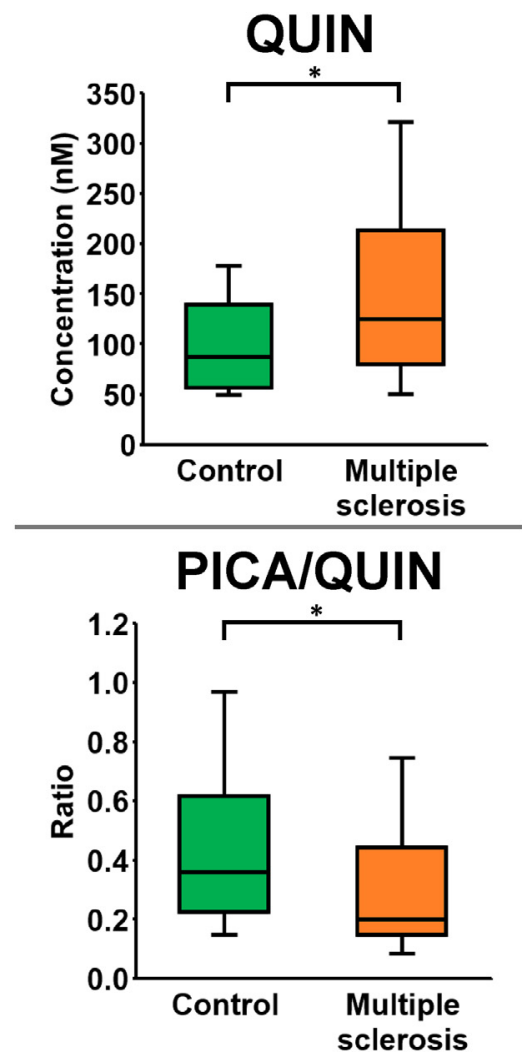

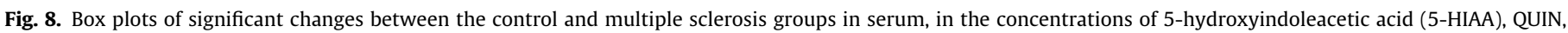

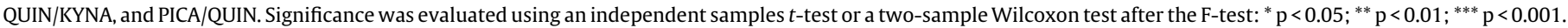

ratio in the multiple sclerosis group than in the control group. The QUIN/KYNA ratio reflects excitotoxicity because excitotoxicity is increasingly favored as the ratio increases. The hypothesis that excitotoxic TRP metabolites can cause neurodegeneration in multiple sclerosis is supported by these data.

The KYNA/KYN ratio (a potential surrogate marker of KAT activity) decreased in the multiple sclerosis group compared with in the control group. This originated from decreased metabolism of the neuroprotective branch of $\mathrm{KP}$, a phenomenon described in other disorders [27].

In addition, the PICA/QUIN ratio, which increased in the multiple sclerosis group, may be a result of the ability of neuroprotective PICA to antagonize QUIN neurotoxicity [28] or may be representative of the inflammatory processes in multiple sclerosis.

In CSF samples, the measured 3-HK concentration significantly increased, which is expected because this neurotoxic metabolite is known to potentiate QUIN-induced excitotoxicity [8]. Moreover, QUIN is involved in the phosphorylation of neurofilaments, the structural components of axons [29]. The increased concentration of neurofilaments in CSF and serum of patients with multiple sclerosis reflects the extent of neuroaxonal damage, as described in a previous study [30].

\section{Conclusion}

We validated a UHPLC-MS/MS method for the simultaneous quantitative estimation of TRP and its 11 most important metabolites derived via both KP and SERO pathways (SERO, KYN, 3-HANA, 5-HIAA, ANA, KYNA, 3-HK, XA, MELA, PICA, and QUIN). The validated chromatographic method is accurate and applicable to human CSF and serum. Although 3 metabolites (3-HK, PICA, and QUIN) were analyzed in derivatized forms, they were assessed together with 9 underivatized metabolites in a single run. The chromatographic method was in silico optimized using DryLab ${ }^{\circledR} 4$. Selectivity, linearity, LOD, LOQ, precision, accuracy, and recovery values upon validation were within the acceptable ranges recommended by the ICH and FDA guidelines [17,18]. The applicability of the chromatographic method was proved by comparing the described concentrations obtained using biological matrices with literature data.

This study provides a reference for the clinical and scientific research of multiple sclerosis. Our results suggest that ratios of different TRP metabolites could be putative biomarkers of this disease. To the best of our knowledge, this is the first study to quantitatively estimate derivatized 3-HK, PICA, and QUIN simultaneously with 9 other TRP metabolites in human serum and CSF.

\section{Declaration of Competing Interest}

The authors declare that they have no known competing financial interests or personal relationships that could have appeared to influence the work reported in this paper.

\section{CRediT authorship contribution statement}

Ferenc Tömösi: Methodology, Investigation, Formal analysis, Writing - original draft, Visualization. Gábor Kecskeméti: Methodology, Investigation, Formal analysis, Writing - original draft. Edina Katalin Cseh: Writing - original draft. Elza Szabó: Resources. Cecília Rajda: Writing - original draft. Róbert Kormány: Data curation, Software. Zoltán Szabó: Formal analysis, Data curation. László Vécsei: Conceptualization, Supervision, Funding acquisition. Tamás Janáky: Conceptualization, Supervision, Funding acquisition, Writing - review \& editing. 


\section{Acknowledgements}

This work was supported by the TUDFO/47138-1/2019ITM, EFOP-3.6.1-16-2016-00008, and GINOP-2.3.2-15-2016-00034 projects. Edina Katalin Cseh was supported by the UNKP-19-3 New National Excellence Program of the Ministry for Innovation and Technology and the EFOP-3.6.3-VEKOP-16-2017-00009.

The authors are grateful to Dr. Imre Molnár, the founder of "Molnár-Institute for Applied Chromatography" for providing us a license to DryLab ${ }^{\circledR} 4$.

\section{Appendix A. Supplementary data}

Supplementary material related to this article can be found, in the online version, at doi:https://doi.org/10.1016/j.jpba.2020. 113246.

\section{References}

[1] L. Vécsei, L. Szalárdy, F. Fülöp, J. Toldi, Kynurenines in the CNS: recent advances and new questions, Nat. Rev. Drug Discov. 12 (2013) 64-82, http:// dx.doi.org/10.1038/nrd3793.

[2] D. Zádori, P. Klivényi, I. Plangár, J. Toldi, L. Vécsei, Endogenous neuroprotection in chronic neurodegenerative disorders: with particular regard to the kynurenines, J. Cell. Mol. Med. 15 (2011) 701-717, http://dx.doi. org/10.1111/j.1582-4934.2010.01237.x.

[3] A.J. Thompson, B.L. Banwell, F. Barkhof, W.M. Carroll, T. Coetzee, G. Comi, J. Correale, F. Fazekas, M. Filippi, M.S. Freedman, K. Fujihara, S.L. Galetta, H.P. Hartung, L. Kappos, F.D. Lublin, R.A. Marrie, A.E. Miller, D.H. Miller, X. Montalban, E.M. Mowry, P.S. Sorensen, M. Tintoré, A.L. Traboulsee, M. Trojano, B.M.J. Uitdehaag, S. Vukusic, E. Waubant, B.G. Weinshenker, S.C. Reingold, J.A. Cohen, Diagnosis of multiple sclerosis: 2017 revisions of the McDonald criteria, Lancet Neurol. 17 (2018) 162-173, http://dx.doi.org/10.1016/S14744422(17)30470-2.

[4] G. Giovannoni, B. Turner, S. Gnanapavan, C. Offiah, K. Schmierer, M. Marta, Is it time to target no evident disease activity (NEDA) in multiple sclerosis? Mult. Scler. Relat. Disord. 4 (2015) 329-333, http://dx.doi.org/10.1016/j. msard.2015.04.006.

[5] B. Fakan, L. Szalardy, L. Vecsei, Exploiting the therapeutic potential of endogenous immunomodulatory systems in multiple sclerosis- special focus on the peroxisome proliferator-activated receptors (PPARs) and the kynurenines, Int. J. Mol. Sci. 20 (2019), http://dx.doi.org/10.3390/ ijms20020426.

[6] S. Aeinehband, P. Brenner, S. Ståhl, M. Bhat, M.D. Fidock, M. Khademi, T. Olsson, G. Engberg, J. Jokinen, S. Erhardt, F. Piehl, Cerebrospinal fluid kynurenines in multiple sclerosis; relation to disease course and neurocognitive symptoms, Brain Behav. Immun. 51 (2016) 47-55, http://dx. doi.org/10.1016/j.bbi.2015.07.016.

[7] K. Rejdak, H. Bartosik-Psujek, B. Dobosz, T. Kocki, P. Grieb, G. Giovannoni, W.A. Turski, Z. Stelmasiak, Decreased level of kynurenic acid in cerebrospinal fluid of relapsing-onset multiple sclerosis patients, Neurosci. Lett. 331 (2002) 63-65, http://dx.doi.org/10.1016/S0304-3940(02)00710-3.

[8] C.K. Lim, A. Bilgin, D.B. Lovejoy, V. Tan, S. Bustamante, B.V. Taylor, A. Bessede, B.J. Brew, G.J. Guillemin, Kynurenine pathway metabolomics predicts and provides mechanistic insight into multiple sclerosis progression, Sci. Rep. 7 (2017), http://dx.doi.org/10.1038/srep41473.

[9] E. Hényková, H.P. Vránová, P. Amakorová, T. Pospíšil, A. Žukauskaitè, M. Vlčková, L. Urbánek, O. Novák, J. Mareš, P. Kaňovský, M. Strnad, Stable isotope dilution ultra-high performance liquid chromatography-tandem mass spectrometry quantitative profiling of tryptophan-related neuroactive substances in human serum and cerebrospinal fluid, J. Chromatogr. A 1437 (2016) 145-157, http://dx.doi.org/10.1016/j.chroma.2016.02.009.

[10] R. Fuertig, A. Ceci, S.M. Camus, E. Bezard, A.H. Luippold, B. Hengerer, LC-MS/MS-based quantification of kynurenine metabolites, tryptophan, monoamines and neopterin in plasma, cerebrospinal fluid and brain, Bioanalysis. 8 (2016) 1903-1917, http://dx.doi.org/10.4155/bio-2016-0111.

[11] W. Zhu, A.P. Stevens, K. Dettmer, E. Gottfried, S. Hoves, M. Kreutz, E. Holler, A.B. Canelas, I. Kema, P.J. Oefner, Quantitative profiling of tryptophan metabolites in serum, urine, and cell culture supernatants by liquid chromatography-tandem mass spectrometry, Anal. Bioanal. Chem. 401 (2011) 3249-3261, http://dx.doi.org/10.1007/s00216-011-5436-y.

[12] P.K. Sahu, N.R. Ramisetti, T. Cecchi, S. Swain, C.S. Patro, J. Panda, An overview of experimental designs in HPLC method development and validation, J. Pharm. Biomed. Anal. 147 (2018) 590-611, http://dx.doi.org/10.1016/j.jpba. 2017.05.006.

[13] S. Fekete, I. Molnár, H.-J. Rieger, R. Kormány. 2018. HPLC method development by QbD compatible resolution modeling (DryLab4), in: Software-assisted method development in high performance liquid chromatography, World Scientific (Europe) see: https://www.worldscientific. com/doi/abs/10.1142/97817; 11-51.

[14] Brain slice electrophysiology | Protocol | Abcam, (2018). https://www.abcam.com/protocols/brain-slice-electrophysiology-videoprotocol (accessed September 17, 2019).

[15] M. Möller, J.L. Du Preez, B.H. Harvey, Development and validation of a single analytical method for the determination of tryptophan, and its kynurenine metabolites in rat plasma, J. Chromatogr. B Analyt. Technol. Biomed. Life Sci. 898 (2012) 121-129, http://dx.doi.org/10.1016/j.jchromb.2012.04.030.

[16] C.E. Teunissen, A. Petzold, J.L. Bennett, F.S. Berven, L. Brundin, M. Comabella, D. Franciotta, J.L. Frederiksen, J.O. Fleming, R. Furlan, R.Q. Hintzen, S.G. Hughes, M.H. Johnson, E. Krasulova, J. Kuhle, M.C. Magnone, C. Rajda, K. Rejdak, H.K. Schmidt, V. van Pesch, E. Waubant, C. Wolf, G. Giovannoni, B. Hemmer, H. Tumani, F. Deisenhammer, A consensus protocol for the standardization of cerebrospinal fluid collection and biobanking, Neurology. 73 (2009) 1914-1922, http://dx.doi.org/10.1212/WNL.0b013e3181c47cc2.

[17] P. Borman, D. Elder, Q2(R1) Validation of Analytical Procedures, in: ICH Qual. Guidel, John Wiley \& Sons, Inc, 2017, pp. 127-166, http://dx.doi.org/10.1002/ 9781118971147, ch5.

[18] FDA CDER, Bioanalytical Method Validation Guidance for Industry, 2018 https://www.fda.gov/files/drugs/published/Bioanalytical-Method-ValidationGuidance-for-Industry.pdf.

[19] H. Trufelli, P. Palma, G. Famiglini, A. Cappiello, An overview of matrix effects in liquid chromatography-mass spectrometry, Mass Spectrom. Rev. 30 (2011) 491-509, http://dx.doi.org/10.1002/mas.20298.

[20] R Core Team, R: A Language and Environment for Statistical Computing, 2019 https://www.r-project.org/.

[21] S. Fanali, R. Haddad, P.C. Poole, M.-L. Riekkola, Derivatization in liquid chromatography, in: Handbook in Separation Science: Liquid Chromatography, 2nd ed., Elsevier, Amsterdam, 2017.

[22] D.H. Chace, D.S. Millington, N. Terada, S.G. Kahler, C.R. Roe, L.F. Hofman, Rapid diagnosis of phenylketonuria by quantitative analysis for phenylalanine and tyrosine in neonatal blood spots by tandem mass spectrometry, Clin. Chem. 39 (1993) 66-71.

[23] C.T.S.D.S. Bell, Mechanisms of interaction responsible for alternative selectivity of fluorinated stationary phases, LCGC North Am. 34 (2016) 92-105.

[24] Chemicalize - Instant Cheminformatics Solutions, (n.d.). https://chemicalize.com/ (accessed November 8, 2019).

[25] DryLab ${ }^{\circledR}$ Publications, 2019. http://molnar-institute.com/fileadmin/user_upload/Publication_List.pdf (accessed November 12, 2019).

[26] L.H. Zhang, H.L. Cai, P. Jiang, H. De Li, L.J. Cao, R.L. Dang, W.Y. Zhu, Y. Deng Simultaneous determination of multiple neurotransmitters and their metabolites in rat brain homogenates and microdialysates by LC-MS/MS, Anal. Methods 7 (2015) 3929-3938, http://dx.doi.org/10.1039/c5ay00308c.

[27] H. Liu, L. Ding, H. Zhang, D. Mellor, H. Wu, D. Zhao, C. Wu, Z. Lin, J. Yuan, D. Peng, The metabolic factor kynurenic acid of kynurenine pathway predicts major depressive disorder, Front. Psychiatry 9 (2018), http://dx.doi.org/10. 3389/fpsyt.2018.00552.

[28] E.Y. Bryleva, L. Brundin, Kynurenine pathway metabolites and suicidality, Neuropharmacology. 112 (2017) 324-330, http://dx.doi.org/10.1016/j. neuropharm.2016.01.034.

[29] P. Pierozan, F. Ferreira, B.O. de Lima, C.G. Fernandes, T.P. Monteforte, N. de C. Medaglia, C. Bincoletto, S.S. Smaili, R. Pessoa-Pureur, The phosphorylation status and cytoskeletal remodeling of striatal astrocytes treated with quinolinic acid, Exp. Cell Res. 322 (2014) 313-323, http://dx.doi.org/10.1016/ j.yexcr.2014.02.024.

[30] G. Disanto, C. Barro, P. Benkert, Y. Naegelin, S. Schädelin, A. Giardiello, C. Zecca, K. Blennow, H. Zetterberg, D. Leppert, L. Kappos, C. Gobbi, J. Kuhle, Serum Neurofilament light: a biomarker of neuronal damage in multiple sclerosis, Ann. Neurol. 81 (2017) 857-870, http://dx.doi.org/10.1002/ana.24954. 